\title{
Ubiquitin-specific peptidase 22 inhibits colon cancer cell invasion by suppressing the signal transducer and activator of transcription 3/matrix metalloproteinase 9 pathway
}

\author{
NING AO, YANYAN LIU, XIAOCUI BIAN, HAILIANG FENG and YUQIN LIU \\ Department of Pathology, Institute of Basic Medical Sciences, Chinese Academy of Medical Sciences \\ and Peking Union Medical College, Tsinghua University, Beijing 100005, P.R. China \\ Received July 1, 2014; Accepted March 10, 2015
}

DOI: $10.3892 / \mathrm{mmr} .2015 .3661$

\begin{abstract}
Colon cancer is associated with increased cell migration and invasion. In the present study, the role of ubiquitin-specific peptidase 22 (USP22) in signal transducer and activator of transcription 3 (STAT3)-mediated colon cancer cell invasion was investigated. The messenger RNA levels of STAT3 target genes were measured by reverse transcription-quantitative polymerase chain reaction, following USP22 knockdown by RNA interference in SW480 colon cancer cells. The matrix metalloproteinase 9 (MMP9) proteolytic activity and invasion potential of SW480 cells were measured by zymography and Transwell assay, respectively, following combined USP22 and STAT3 short interfering (si)RNA treatment or STAT3 siRNA treatment alone. Similarly, a cell counting kit- 8 assay was used to detect the proliferation potential of SW480 cells. The protein expression levels of USP22, STAT3 and MMP9 were detected by immunohistochemistry in colon cancer tissue microarrays (TMAs) and the correlation between USP22, STAT3 and MMP9 was analyzed. USP22/STAT3 co-depletion partly rescued the MMP9 proteolytic activity and invasion of SW480 cells, compared with that of STAT3 depletion alone. However, the proliferaton of USP22/STAT3si-SW480 cells was decreased compared with that of STAT3si-SW480 cells. USP22 expression was positively correlated with STAT3 and MMP9 expression in colon cancer TMAs. In conclusion, USP22 attenuated the invasion capacity of colon cancer cells by inhibiting the STAT3/MMP9 signaling pathway.
\end{abstract}

Correspondence to: Professor Yuqin Liu, Department of Pathology, Institute of Basic Medical Sciences, Chinese Academy of Medical Sciences and Peking Union Medical College, Tsinghua University, 5 Dong Dan San Tiao, Beijing 100005, P.R. China E-mail: ccc@pumc.edu.cn

Key words: ubiquitin-specific peptidase 22, signal transducer and activator of transcription 3, matrix metalloproteinase 9, invasion, colon cancer

\section{Introduction}

Cancer remains a major public health issue worldwide, despite significant advances in elucidating cancer molecular biology and treatment. Colon cancer is the third most common cause of cancer-associated mortality, while the molecular mechanisms underlying the development and progression of colon cancer remain poorly understood (1).

Ubiquitin-specific peptidase 22 (USP22) is one of an 11-gene Polycomb/cancer stem cell signature that may be able to powerfully predict the therapeutic outcome of individual cancer patients (2). USP22 expression is correlated with colorectal cancer progression and therapy failure (3). In addition, USP22 may regulate the cell cycle via the BMI-1-mediated INK4a/ARF and glycogen synthase kinase 3/Akt signaling pathways in HCT116 colon cancer cells $(4,5)$.

Signal transducer and activator of transcription (STAT) proteins are a family of latent cytoplasmic transcription factors, which mediate cellular responses to cytokines and growth factors $(6,7)$. The activation of STAT3 by phosphorylation is mediated by cytoplasmic and growth factor receptor tyrosine kinases (8). STAT3 is involved in the oncogenesis of colorectal cancer, promoting the proliferation and invasion of colon cancer cells $(9,10)$.

USP22-mediated sirtuin 1 (SIRT1) deubiquitination in HCT116 cells leads to decreased p53 acetylation and the suppression of p53-mediated apoptosis (11). A previous study by our group demonstrated that USP22 inhibited STAT3 acetylation and the activation of STAT3 target genes MMP 9 and TWIST by deubiquitination of SIRT1 in HT29 and SW480 colon cancer cells (12). In another previous study by our group, MMP9 and TWIST messenger (m)RNA expression was evaluated following combined USP22 and STAT3 short interfering (si)RNA treatment, compared with STAT3 siRNA alone in colon cancer cells. USP22/STAT3 co-depletion partly reduced the MMP9 and TWIST mRNA levels, induced by STAT3 depletion alone. Although USP22 suppresses the STAT3 signaling pathway in colon cancer cells, whether USP22 modulates STAT3-mediated colon cancer function in particular has remained to be elucidated.

In the present study, the proliferation and invasion potential of SW480 cells following STAT3 or USP22/STAT3 
knockdown by RNA interference was evaluated. In addition, the associations between USP22, STAT3 and MMP9 were investigated with a colon cancer tissue microarray (TMA). The present study aimed to identify a novel mechanism through which USP22 may affect colon cancer cell invasion.

\section{Materials and methods}

Cell culture. The SW480 colon cancer cell line (adenocarcinoma), Iscove's modified Dulbecco's medium (IMDM) and $10 \%$ fetal bovine serum (FBS) used in the present study were obtained from the Cell Resource Center, Institute of Basic Medical Sciences, Chinese Academy of Medical Sciences, Peking Union Medical College (CRC-PUMC; Beijing, China), and were authenticated as mycoplasma-free by short tandem-repeat polymerase chain reaction (PCR). Cells were cultured under the conditions recommended by the CRC-PUMC (http://cellresource.cn). The SW480 cell line was maintained in IMDM containing $10 \%$ FBS. All cell lines were grown under $5 \% \mathrm{CO}_{2}$ at $37^{\circ} \mathrm{C}$ in a humidified environment.

RNA silencing. The siRNA method was used to knock-down USP22 expression in SW480 cells. USP22 siRNA was obtained from Guangzhou RiboBio Co., Ltd (Guangzhou, China), and the sequence was as follows: 5'-GGAGAAAGAUCACCU CGAADTDT-3'. STAT3 siRNA was obtained from Invitrogen Life Technologies (Carlsbad, CA, USA) and the sequence was: 5'-GGAAAGAAGGAAUAUCUCADTDT-3'. Ncontrol_05815 (NCsi; RiboBio Co., Ltd) was used as a negative control for all siRNA experiments. To select the most efficient siRNA, SW480 cells were transfected with the siRNAs using Lipofectamine ${ }^{\circledR} 2000$ (Invitrogen Life Technologies), according to the manufacturer's instructions. Briefly, the SW480 cells were seeded without antibiotics at $1 \times 10^{6}$ cells in a $25 \mathrm{~cm}^{2}$ culture bottle. Prior to transfection, the cells were washed with phosphate-buffered saline (PBS). The cells were then divided into three groups: Those transfected with STAT3 siRNA, those transfected with both USP22 siRNA and STAT3 siRNA, and those transfected with negative control siRNA. The cell culture medium was changed after $6 \mathrm{~h}$. The cells were incubated an additional $48 \mathrm{~h}$, after which functional siRNA was measured by reverse transcription (RT)-PCR.

RNA isolation and RT-PCR. Total RNA was extracted using TRIzol reagent (Life Technologies, Grand Island, NY, USA). RT was performed using a PrimeScript RT Master Mix kit (Takara Biotechnology Co. Ltd., Dalian, China). The complementary DNA products were subjected to PCR using a SYBR Premix Ex TaqTM II kit (Takara Biotechnology Co. Ltd.). PCR was performed using a StepOnePlus Real-Time PCR system (Applied Biosystems Life Technologies, Carlsbad, CA, USA) using the following program: $95^{\circ} \mathrm{C}$ for $10 \mathrm{~min}$, followed by 40 cycles of $95^{\circ} \mathrm{C}$ for $15 \mathrm{sec}$ and $60^{\circ} \mathrm{C}$ for $1 \mathrm{~min}$. Each sample was analyzed in triplicate. GAPDH mRNA was quantified and used as an endogenous control. The RNA expression was determined using the $2^{-\Delta \Delta \mathrm{Ct}}$ method (13). The primer sequences used in the present study are shown in Table I.

Zymography. MMP2 and MMP9 proteolytic activity of the supernatants was determined by gelatin zymography. At
$24 \mathrm{~h}$ post-siRNA transfection, the transfection medium was removed and cells were incubated in serum-free medium for a further $48 \mathrm{~h}$ at $37^{\circ} \mathrm{C}$. Serum-free supernatants were then subjected to $10 \%$ SDS-PAGE containing $0.1 \%$ gelatin (Beyotime Institute of Biotechnology, Haimen, China). Gels were incubated twice for $45 \mathrm{~min}$ at room temperature in $2.5 \%$ Triton X-100 (Qxbio, Beijing, China), and then incubated in activation buffer $\left(50 \mathrm{mmol} / 1\right.$ Tris- $\mathrm{Cl} \mathrm{pH} \mathrm{8.0,10} \mathrm{mmol} / 1 \mathrm{CaCl}_{2}$, $50 \mathrm{mmol} / \mathrm{l} \mathrm{NaCl}$ ) for $42 \mathrm{~h}$ at $37^{\circ} \mathrm{C}$. Subsequently, gels were stained with $0.05 \%$ Coomassie Brilliant Blue R-250 (Beyotime Institute of Biotechnology) for $3 \mathrm{~h}$ and de-stained sequentially in wash buffers I (10\% acetic acid, $30 \%$ methanol), II (10\% acetic acid, 20\% methanol) and III (5\% acetic acid, 10\% methanol) for $0.5,1$ and $2 \mathrm{~h}$ at $37^{\circ} \mathrm{C}$, respectively. Regions of gelatinolytic activity were detected as transparent bands on a blue background. The relative molecular weight of each band was determined using protein standards (Pierce Biotechnology, Inc., Rockford, IL, USA). Areas of protease activity appeared as unstained bands against a blue background. Images were assessed by ImageJ 1.44 (National Institutes of Health, Bethesda, MA, USA).

In vitro invasion assays. In vitro invasion was measured using an in vitro Transwell assay. The 24-well Transwell polycarbonate membrane inserts with $8.0-\mathrm{mm}$ pores (Costar, Cambridge, MA, USA) were coated with Matrigel (1:8, $70 \mathrm{ul}$; 354277; BD Biosciences, San Jose, CA, USA) at the bottom. SW480 cells $\left(1 \times 10^{6}\right)$ were plated in the upper chamber in $0.2 \mathrm{ml}$ IMDM supplemented with $1 \%$ bovine serum albumin (BSA; Origene Technologies, Inc., Beijing, China), and the lower chamber was filled with $0.5 \mathrm{ml}$ IMDM containing $10 \%$ BSA. Cells were incubated for $48 \mathrm{~h}$, and cells remaining on the upper side of the membrane were gently removed with wet cotton swabs. The invading cells on the lower surface of the membrane were stained with crystal violet staining solution (C0121; Beyotime Institute of Biotechnology) for 30 min prior to washing. The number of cells in nine randomly selected microscopic fields per well (magnification, x20) was counted under an Olympus IX51 microscope (Olympus Corp., Tokyo, Japan). All experiments were repeated in triplicate.

Cell counting kit-8 (CCK8) cell viability assay. Cell viability was assessed by a CCK8 assay (Dojindo Molecular Technologies, Inc., Kumamoto, Japan). SW480 cells ( $2 \times 10^{3}$ cells/well) were plated in 96-well plates, and following $24 \mathrm{~h}$ of culture at $37^{\circ} \mathrm{C}$, the cells were treated with STAT3 siRNA or USP22/STAT3 siRNA. Following incubation with clotrimazole for 24,48 or $72 \mathrm{~h}$ at $37^{\circ} \mathrm{C}$, the CCK8 reagent was added to each well and cells were incubated for $2 \mathrm{~h}$ at $37^{\circ} \mathrm{C}$. Subsequently, the absorbance (optical density) at $450 \mathrm{~nm}$ was measured using a Synergy 4 reader (BioTek, Winooski, VT, USA). All experiments were repeated in triplicate.

Immunohistochemistry. TMAs were designed as described previously (14), using three $0.4 \mathrm{~mm}$ tissue cores per case, taken from formalin-fixed, paraffin-embedded archival biopsy blocks (Shanghai Biochip Co Ltd, Shanghai, China). TMAs were fixed in $4 \%$ paraformaldehyde in PBS for $30 \mathrm{~min}$ and preincubated in blocking buffer (PBS containing 10\% normal rabbit serum) for $30 \mathrm{~min}$. The TMAs were then incubated at $4^{\circ} \mathrm{C}$ overnight 
Table I. Primers used for reverse transcription-quantitative polymerase chain reaction analysis.

\begin{tabular}{ll}
\hline Primer & \multicolumn{1}{c}{ Sequence } \\
\hline USP22 & S: 5'-GACAACTGGAAGCAGAACC-3' \\
& A: 5'-CGTACATCAGATCAATGGC-3' \\
MMP2 & S: 5'-CTTCCAAGTCTGGAGCGATGT-3' \\
& A: 5'-TACCGTCAAAGGGGTATCCAT-3' \\
TIMP1 & S: 5'-AGTGGCACTCATTGCTTGTG-3' \\
& A: 5'-GCAGGATTCAGGCTATCTGG-3' \\
Hif-1 $\alpha$ & S: 5'-CCGAGGAAGAACTATGAA-3' \\
& A: 5'-GTTGGTTACTGTTGGTATC-3' \\
VEGF & S: 5'-ACACATTGTTGGAAGAAGCAGCCC-3' \\
& A: 5'-AGGAAGGTCAACCACTCACACACA-3' \\
MMP9 & S: 5'-TACCGAGAGAAAGCCTATT-3' \\
& A: 5'-CACCTGGTTCAACTCACT-3' \\
TWIST & S: 5'-GGAGTCCGCAGTCTTACGAG-3' \\
& A: 5'-TCTGGAGGACCTGGTAGAGG-3' \\
GAPDH & S: 5'-AGGTCGGTGTGAACGGATTTG-3' \\
& A: 5'-TGTAGACCATGTAGTTGAGGTCA-3' \\
\end{tabular}

S, Sense; A, Antisense.

Table II. Correlation between expression of USP22, STAT3 and MMP9 in colon cancer tissue microarray ${ }^{\mathrm{a}}$.

\begin{tabular}{lccccc}
\hline Protein & - & + & ++ & +++ & $\overline{\mathrm{X}} \pm \mathrm{S}$ \\
\hline USP22 & 3 & 7 & 13 & 16 & $2.08 \pm 0.96$ \\
STAT3 & 2 & 7 & 5 & 25 & $2.36 \pm 0.96$ \\
MMP9 & 1 & 7 & 10 & 21 & $2.31 \pm 0.86$ \\
\hline
\end{tabular}

aPearson correlation between USP22 and STAT3 $(\mathrm{r}=0.457, \mathrm{P}<0.01)$, USP22 and MMP9 ( $\mathrm{r}=0.385, \mathrm{P}<0.05)$, STAT3 and MMP9 ( $\mathrm{r}=0.785$, $\mathrm{P}<0.01)$. s, standard deviation; USP22, ubiquitin-specific peptidase 22; STAT3, signal transducer and activator of transcription 3; MMP9, matrix metalloproteinase 9 .

with the primary antibodies. Monoclonal anti-human USP22 antibody $(1: 200 ; 3813-1)$ was purchased from Epitomics (Burlingame, CA, USA), monoclonal anti-STAT3 antibody (1:300; 4904S) was obtained from Cell Signaling Technology, Inc. (Danvers, MA, USA), and polyclonal anti-MMP9 antibody (1:300; BS1241) was purchased from Bioworld (Beijing, China). After washing with PBS, the TMAs were incubated with horseradish peroxidase-labeled rabbit anti-goat immunoglobulin G secondary antibody (1:200; Origene Technologies, Inc.) at $37^{\circ} \mathrm{C}$ for $1 \mathrm{~h}$. After washing with PBS, bound antibody was detected with the substrate reagents from HRP-DAB System of Tissue Staining kit (Origene Technologies, Inc.). USP22, STAT3 and MMP9-positive samples were defined as those exhibiting staining. Each case was evaluated by estimating the percentage and intensity of tumor cells displaying a nuclear or cytoplasmic staining pattern. The USP22 and

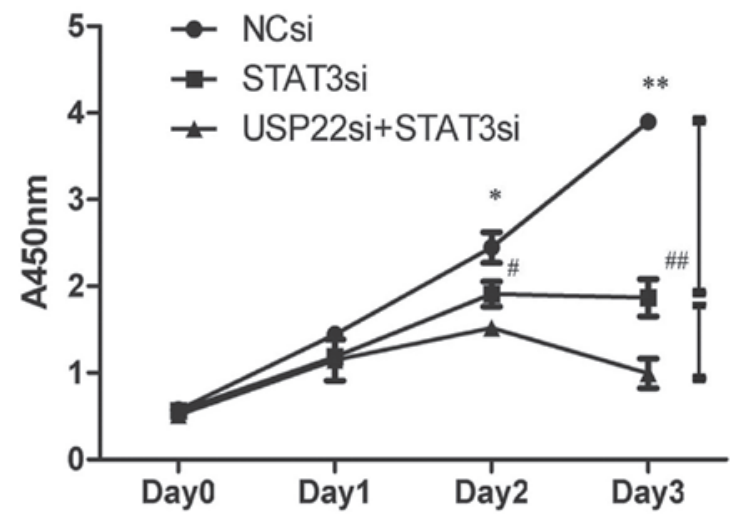

Figure 1. USP22 promotes STAT3-mediated proliferation of SW480 cells. Proliferation potential of SW480 cells transfected with NCsi, STAT3si or a combination of USP22si/STAT3si was evaluated using a Cell counting kit- 8 assay. Values are presented as the mean \pm standard deviation. ${ }^{*} \mathrm{P}<0.01$, ${ }^{* *} \mathrm{P}<0.001$, compared with STAT3si; ${ }^{\#} \mathrm{P}<0.05$, ${ }^{\# \#} \mathrm{P}<0.001$, compared with USP22si+STAT3si. USP22, ubiquitin-specific peptidase 22; STAT3, signal transducer and activator of transcription 3; NC, normal control; si, short interfering RNA.

STAT3 staining patterns were categorized into four groups: 0 , no nuclear staining; $1+, \leq 25 \%$ staining of tumor cells; $2+$, $26-50 \%$ staining of tumor cells and $3+, 50-100 \%$ staining of tumor cells. MMP9 staining patterns were categorized into four groups: 0 , no cytoplasmic staining; $1+, \leq 25 \%$ staining of tumor cells; $2+, 26-50 \%$ staining of tumor cells and $3+$, $50-100 \%$ staining of tumor cells. Categorizing of the USP22, STAT3 and MMP9 staining was conducted by two independent investigators in the Department of Pathology (Institute of Basic Medical Sciences, Chinese Academy of Medical Sciences and Peking Union Medical College, Tsinghua University, Beijing, China), who were blinded to the clinicopathological data, with a consensus reached in all cases.

Statistical analysis. Data are expressed as the mean \pm standard deviation. Statistical analyses to compare pairs of experimental groups were performed using the Student's t-test or two-sample t-test with SPSS version 17.0 statistics software (SPSS, Inc., Chicago, IL, USA) . Correlations between immunohistochemical expression and the clinicopathological characteristics were analyzed by Pearson's $\chi^{2}$ tests. $\mathrm{P}<0.05$ was considered to indicate a statistically significant difference.

\section{Results}

USP22 promotes STAT3-mediated proliferation of SW480 cells. USP22 suppresses STAT3 acetylation by deubiquitinating SIRT1 (12). To detect whether USP22 influenced STAT3-mediated proliferation of SW480 cells, a CCK8 assay was performed. The proliferation capacity of USP22/STAT3si-SW480 cells was significantly decreased compared with that of STAT3si-SW480 cells. In addition, SW480 cell proliferation was inhibited following treatment with STAT3 siRNA compared with that of the control (Fig. 1).

USP22 suppresses STAT3-mediated invasion of SW480 cells. Whether USP22 influenced STAT3-mediated SW480 cell invasion was evaluated by Transwell assay. The results indicated 

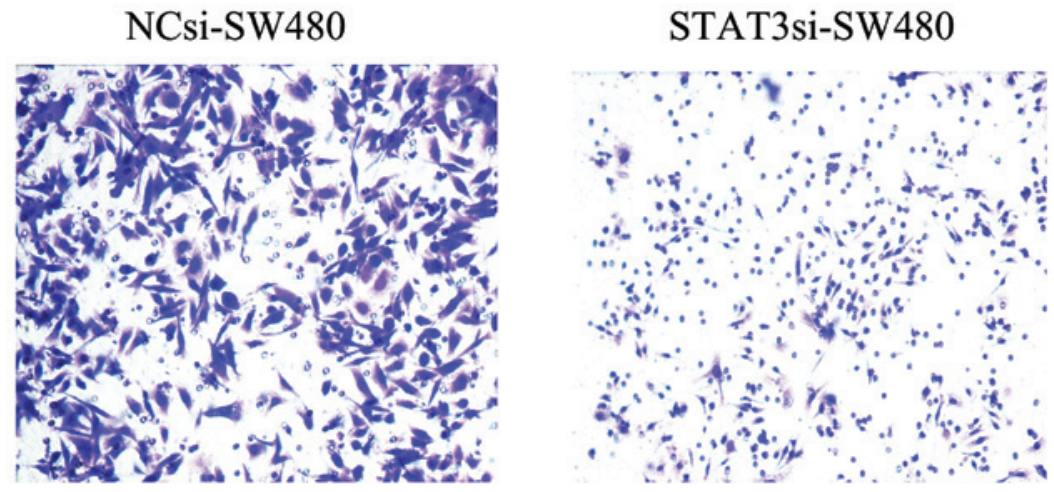

\section{USP22+STAT3si-SW480}
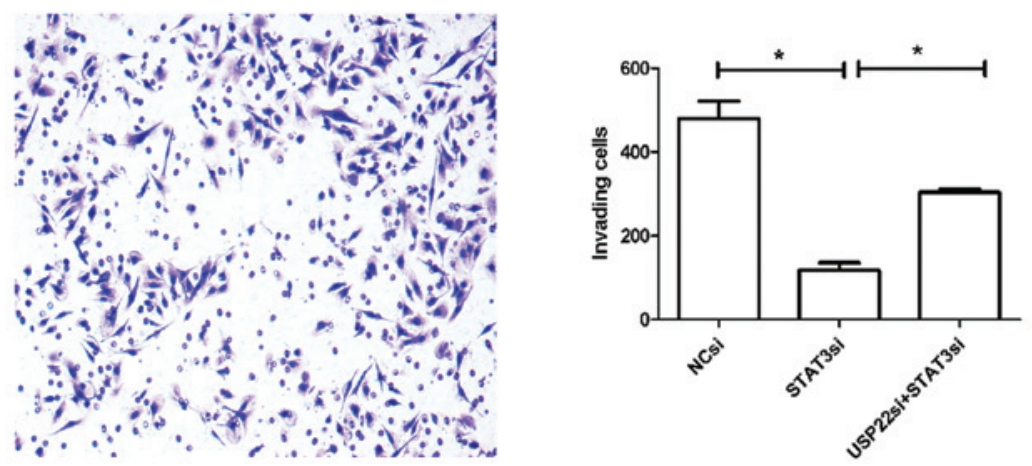

Figure 2. USP22 suppresses STAT3-mediated invasion of SW480 cells. Invasion potential of SW480 cells transfected with NCsi, STAT3si or a combination of USP22si/STAT3si was evaluated using a Transwell assay. Representative photomicrographs of invading cells and the number of invading cells were analyzed (magnification, $\mathrm{x} 40$; staining, crystal violet). Values are presented as the mean \pm standard deviation; ${ }^{*} \mathrm{P}<0.01$. USP22, ubiquitin-specific peptidase 22; STAT3, signal transducer and activator of transcription 3; NC, normal control; si, short interfering RNA.
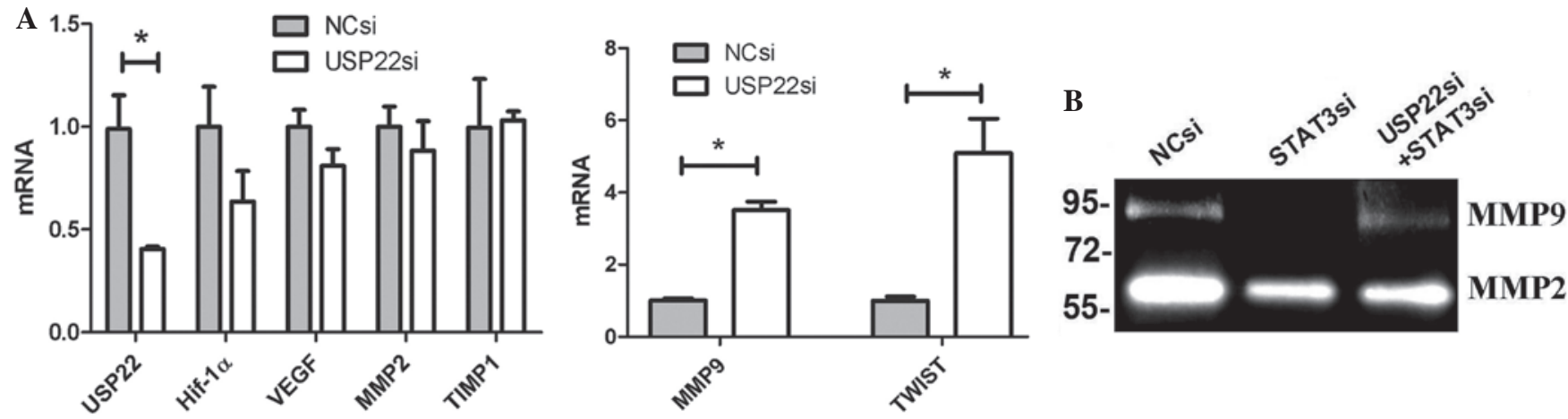

Figure 3. USP22 inhibits STAT3 downstream target genes MMP9 and TWIST in SW480 cells. (A) Total RNA was isolated from SW480 cells transfected with NCsi or USP22si. The mRNA levels of USP22 and STAT3 target genes (Hifl- $\alpha, V E G F, M M P 2, T I M P 1, M M P 9$, TWIST) were determined by reverse transcription polymerase chain reaction, ${ }^{*} \mathrm{P}<0.01$. (B) A zymography assay was used to evaluate the proteolytic activity of MMP2 and MMP9 in the SW480 cells transfected with NCsi, STAT3si and a combination of USP22/STAT3 siRNA. USP22, ubiquitin-specific peptidase 22; STAT3, signal transducer and activator of transcription 3; NC, normal control; si, short interfering; mRNA, messenger RNA; MMP, matrix metalloproteinase.

that the invasion potential of USP22/STAT3si-SW480 cells was increased significantly compared with that of STAT3si-SW480 cells, and SW480 cell invasion was inhibited following treatment with STAT3 siRNA compared with that of the control (Fig. 2).

USP22 inhibits the STAT3-MMP9 pathway in SW480 cells. To examine the mechanism by which USP22 modulates STAT3-mediated cell invasion, the effects of USP22 knockdown on transcripts of the STAT3 target genes Hif-1 $\alpha, V E G F$, MMP2, TIMP1, MMP9 and TWIST were investigated (15-19).
MMP9 and TWIST mRNA levels were significantly increased in USP22si-SW480 cells compared with those of the control cells, as previously described (12); however the other target genes remained unaffected (Fig. 3A). A gelatin zymography assay was used to detect the activity of MMP2 and MMP9 following USP22/STAT3 siRNA and STAT3 siRNA treatment. As shown in Fig. 3B, the activity of MMP2 was decreased following treatment of SW480 cells with STAT3 siRNA or USP22/STAT3 siRNA. STAT3 knockdown reduced MMP9 activity, an effect which was partly rescued by USP22/STAT3 knockdown in SW480 cells. 


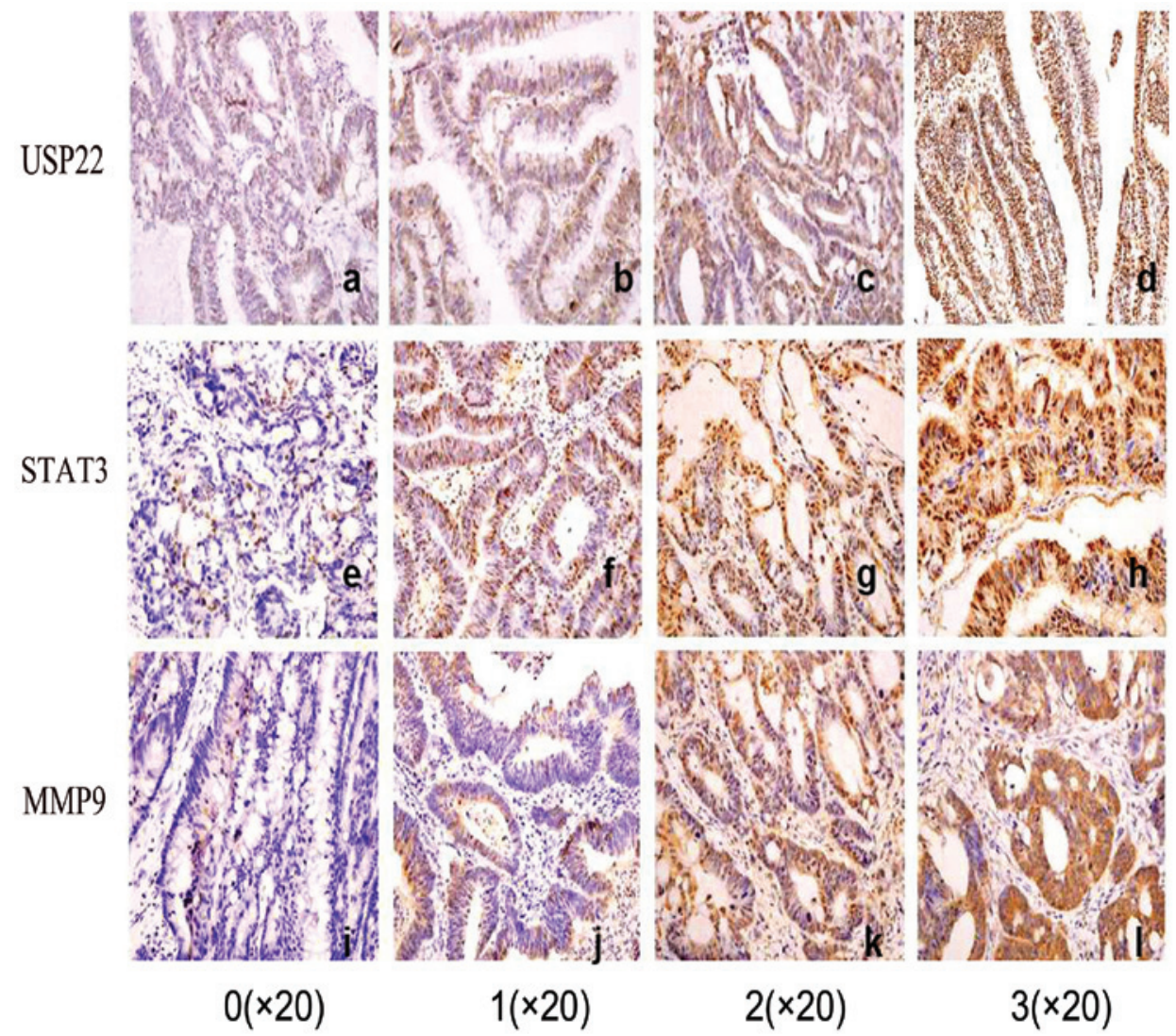

Figure 4. Representative expression of USP22, STAT3 and MMP9 in colon cancer tissues on a tissue microarray slide. USP22 expression (a) 0 staining, (b) 1+ staining, (c) 2+ staining and (d) 3+staining. STAT3 expression (e) 0 staining, (f) 1+ staining, (g) 2+ staining and (h) 3+ staining. MMP9 expression (i) 0 staining, (j) 1+ staining, (k) 2+ staining and (1) 3+ staining (magnification, x20). USP22, ubiquitin-specific peptidase 22; STAT3, signal transducer and activator of transcription 3; MMP9, matrix metalloproteinase 9.

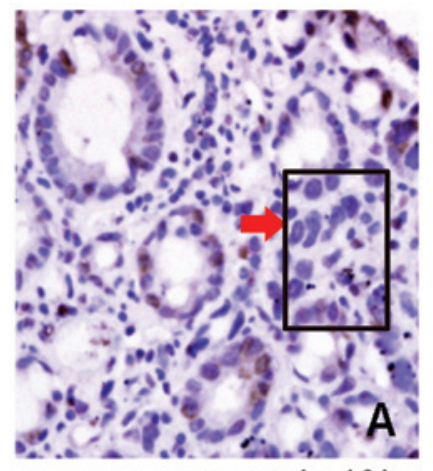

USP22 $(\times 40)$

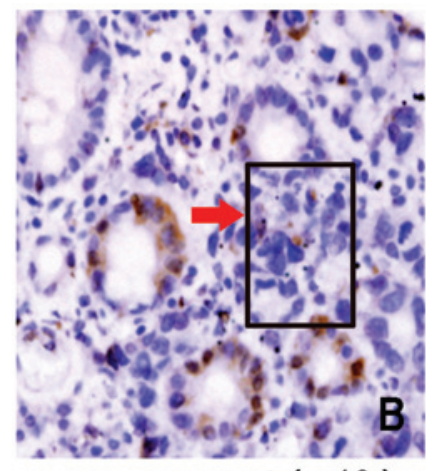

MMP9 $(\times 40)$

Figure 5. At the colon cancer invasion front USP22 expression is low and MMP9 expression is high. (A) USP22 expression was low (red arrow) at the colon cancer invasion front. (B) MMP9 expression was high (red arrow) in colon cancer invasion front.

Analysis of correlation between USP22, STAT3 and MMP9 expression in a colon cancer TMA. To verify the association between USP22, STAT3 and MMP9 in colon cancer, immunohistochemical analysis was performed on a colon cancer TMA $(n=39)$. The results revealed that USP22 and STAT3 staining was localized within the nuclei, and MMP9 staining was localized in the cytoplasm of colorectal epithelial cells (Fig. 4). USP22, STAT3 and MMP9 expression levels were higher in poorly differentiated colorectal adenocarcinoma, as compared

with in the well-differentiated counterparts. As shown in Table II, USP22 exhibited moderate correlation with STAT3 expression $(\mathrm{r}=0.457, \mathrm{P}<0.01)$ and low correlation with MMP9 expression $(\mathrm{r}=0.385, \mathrm{P}<0.05)$. There was a strong correlation detected between STAT3 and MMP9 ( $\mathrm{r}=0.785, \mathrm{P}<0.01)$.

USP22 and MMP9 expression at the colon cancer invasion front. USP22 and MMP9 expression at the colon cancer invasion front was evaluated and the results indicated that USP22 expression was relatively low, while MMP9 expression was higher (Fig. 5).

\section{Discussion}

USP22 has significant roles in mediating colon cancer growth and progression. Liu et al (5) demonstrated that USP22 knockdown in HCT116 cells resulted in the inhibition of cellular proliferation, the accumulation of cells in the $\mathrm{G}_{1}$ phase and repression of BMI-1, c-Myc and p53, accompanied by the upregulation of p16INK4a and p14ARF (4). These results confirmed the critical role of USP22 in the formation and growth of colon cancer cells through the Rb (via c-Myc and BMI-1/p16INK4a) and p53 (via BMI-1/p14ARF) pathways. In the present study, USP22 was found to enhance the STAT3-mediated proliferation potential of SW480 cells. This phenomenon suggested that USP22 may functionally participate in the proliferation of colon cancer cells. 
In a previous study by our group, USP22/SIRT1/STAT3 proteins were found to be present in a single protein complex (12). USP22 downregulated STAT3 acetylation by deubiquitination of SIRT1. In addition, USP22/STAT3 knockdown partly rescued the reduced STAT3 acetylation, as well as $M M P 9$ and TWIST mRNA levels, induced by STAT3 knockdown in colon cancer cells. These results suggested that USP22 may inhibit STAT3 transcription activation by regulating STAT3 acetylation in colon cancer cells. In the present study, it was demonstrated that STAT3 depletion reduced the invasion potential of SW480 cells, an effect which was rescued by USP22/STAT3 co-depletion. These data suggested that USP22 modulates STAT3-mediated SW480 cell invasion and that the association between USP22 and STAT3 may be involved in tumor invasion.

To elucidate the mechanism by which USP22 regulates STAT3-mediated invasion, the effect of USP22 depletion on STAT3-dependent target genes associated with the invasion of tumor cells was examined. The results indicated that $M M P 9$ and TWIST mRNA expression levels were significantly enhanced, as previously described (12), whereas $M M P 2$ and TIMP1 mRNA expression was not significantly altered in USP22si-SW480 cells. STAT3 knockdown-induced MMP9 proteolytic activity and SW480 cell invasion were reduced by the combined knockdown of STAT3 and USP22, further supporting the hypothesis that USP22 may suppress the STAT3-mediated invasion of colon cancer cells by regulating MMP9.

MMPs also have significant roles in mediating tumor invasion and metastasis. Curran et al demonstrated that MMPs were associated with colon cancer progression (20). Atanassov et al (21) observed that FBP1, as a protein with abundant ubiquitination upon USP22 ablation, ensures the activation of target loci, including MMP1 in normal human fibroblasts and HeLa cells. In the present study, USP22 was demonstrated to regulate the invasion potential of SW480 cells by inhibiting MMP9. These results further suggested that USP22 may be involved in tumor invasion by regulating MMPs.

Angiogenesis, defined as the formation of new blood vessels out of existing ones, is an essential prerequisite for tumor growth, progression and metastasis (22). Tumor vascularization, known as the 'angiogenic switch', occurs as a result of imbalanced expression and function of pro-angiogenic factors. Amongst the angiogenic factors involved in regulating tumor angiogenesis, the best known are hypoxia-inducible factorl- $\alpha$ (Hif1- $\alpha$ ) and vascular endothelial growth factor (VEGF) (23). The results of the present study indicated that USP22 depletion did not alter Hif- $1 \alpha$ or VEGF mRNA expression in SW480 cells, suggesting that USP22 may not be involved in colon cancer angiogenesis.

USP22 was found to be associated with certain types of cancer progression and therapy failure. For example, Liu et al (24) demonstrated that USP22 was associated with certain clinicopathological variables, including gross features, differentiation, $\mathrm{pT}$ classification, $\mathrm{pN}$ classification, $\mathrm{pM}$ classification, American Joint Committee of Cancer stage and shorter disease-specific survival in colorectal carcinoma. USP22 mRNA and protein expression levels were significantly higher in primary colon cancer than those in paired non-cancerous tissues (3). The results of the present study indicated that USP22, STAT3 and MMP9 were overexpressed in the majority of primary colon cancer TMAs. Furthermore, statistical analysis of direct correlations indicated that USP22 had a significant positive correlation with STAT3 $(r=0.457$, $\mathrm{P}<0.01)$ and MMP9 $(\mathrm{r}=0.385, \mathrm{P}<0.05)$; and that STAT3 was correlated with MMP9 expression $(\mathrm{r}=0.785, \mathrm{P}<0.01)$ in colon cancer TMAs. Although a positive correlation between USP22, STAT3 and MMP9 was identified, the colon cancer invasion front was found to exhibit low expression levels of USP22, but high expression levels of MMP9, suggesting that USP22 may inhibit MMP9 in colon cancer cell invasion. It was therefore suggested that the function of USP22 varies amongst colon cancer cells, with high expression in colon cancer tissues. Therefore, therapy targeting USP22 inhibition may not be an effective method for the treatment of colon cancers.

\section{References}

1. Siegel R, Ward E, Brawley O and Jemal A: Cancer statistics, 2011: the impact of eliminating socioeconomic and racial disparities on premature cancer deaths. CA Cancer J Clin 61: 212-236, 2011.

2. Glinsky GV: Genomic models of metastatic cancer: functional analysis of death-from-cancer signature genes reveals aneuploid, anoikis-resistant, metastasis-enabling phenotype with altered cell cycle control and activated Polycomb Group (PcG) protein chromatin silencing pathway. Cell Cycle 5: 1208-1216, 2006.

3. Liu YL, Yang YM, Xu H and Dong XS: Aberrant expression of USP22 is associated with liver metastasis and poor prognosis of colorectal cancer. J Surg Oncol 103: 283-289, 2011.

4. Xu H, Liu YL, Yang YM and Dong XS: Knock-down of ubiquitin- specific protease 22 by micro-RNA interference inhibits colorectal cancer growth. Int J Colorectal Dis 27: 21-30, 2012.

5. Liu YL, Jiang SX, Yang YM, et al: USP22 acts as an oncogene by the activation of BMI-1-mediated INK4a/ARF pathway and Akt pathway. Cell Biochem Biophys 62: 229-235, 2012.

6. Darnell JE Jr, Kerr IM and Stark GR: Jak-STAT pathways and transcriptional activation in response to IFNs and other extracellular signaling proteins. Science 264: 1415-1421, 1994.

7. Zhong Z, Wen Z and Darnell JE Jr: Stat3: a STAT family member activated by tyrosine phosphorylation in response to epidermal growth factor and interleukin-6. Science 264: 95-98, 1994.

8. Dauer DJ, Ferraro B, Song L, et al: Stat 3 regulates genes common to both wound healing and cancer. Oncogene 24: 3397-3408, 2005.

9. Wei X, Wang G, Li W, et al: Activation of the JAK-STAT3 pathway is associated with the growth of colorectal carcinoma cells. Oncol Rep 31: 335-341, 2014.

10. Tsareva SA, Moriggl R, Corvinus FM, et al: Signal transducer and activator of transcription 3 activation promotes invasive growth of colon carcinomas through matrix metalloproteinase induction. Neoplasia 9: 279-291, 2007.

11. Lin Z, Yang H, Kong Q, et al: USP22 antagonizes p53 transcriptional activation by deubiquitinating Sirt1 to suppress cell apoptosis and is required for mouse embryonic development. Mol Cell 46: 484-494, 2012.

12. Ao N, Liu Y, Feng H, et al: Ubiquitin-specific peptidase USP22 negatively regulates the STAT signaling pathway by deubiquitinating SIRT1. Cell Physiol Biochem 33: 1863-1875, 2014.

13. Chen C, Ridzon D, Broomer AJ, et al: Real-time quantification of microRNAs by stem-loop RT-PCR. Nucleic Acids Res 33: e179, 2005.

14. Kononen J, Bubendorf L, Kallioniemi A, et al: Tissue microarrays for high-throughput molecular profiling of tumor specimens. Nat Med 4: 844-847, 1998.

15. Alvarez JV and Frank DA: Genome-wide analysis of STAT3 target genes: elucidating the mechanism of STAT-mediated oncogenesis. Cancer Biol Ther 3: 1045-1050, 2004.

16. Bugno M, Graeve L, Gatsios P, et al: Identification of the interleukin-6/oncostatin $\mathrm{M}$ response element in the rat tissue inhibitor of metalloproteinases-1 (TIMP-1) promoter. Nuc Acids Res 23: 5041-5047, 1995. 
17. Cheng GZ, Zhang WZ, Sun M, et al: Twist is transcriptionally induced by activation of STAT3 and mediates STAT3 oncogenic function. J Biol Chem 283: 14665-14673, 2008.

18. Niu G, Briggs J, Deng J, et al: Signal transducer and activator of transcription 3 is required for hypoxia-inducible factor-1alpha RNA expression in both tumor cells and tumor-associated myeloid cells. Mol Cancer Res 6: 1099-1105, 2008.

19. Niu G, Wright KL, Huang M, et al: Constitutive Stat3 activity up-regulates VEGF expression and tumor angiogenesis. Oncogene 21: 2000-2008, 2002.

20. Curran S, Dundas SR, Buxton J, et al: Matrix metalloproteinase/tissue inhibitors of matrix metalloproteinase phenotype identies poor prognosis colorectal cancers. Clin Cancer Res 10: 8229-8234, 2004.
21. Atanassov BS and Dent SY: USP22 regulates cell proliferation by deubiquitinating the transcriptional regulator FBP1. EMBO Rep 12: 924-930, 2011.

22. Griffioen AW and Molema G: Angiogenesis: potentials for pharmacologic intervention in the treatment of cancer, cardiovascular diseases, and chronic inflammation. Pharmacol Rev 52: 237-268, 2000.

23. Ahluwalia A and Tarnawski AS: Critical role of hypoxia sensor-HIF-1 $\alpha$ in VEGF gene activation. Implications for angiogenesis and tissue injury healing. Curr Med Chem 19: 90-97, 2012.

24. Liu YL, Yang YM, Xu H and Dong XS: Increased expression of ubiquitin-specific protease 22 can promote cancer progression and predict therapy failure in human colorectal cancer. J Gastroenterol Hepatol 25: 1800-1805, 2010. 